\title{
Sagittal Split Ramus Osteotomy in the Absence of Bone Marrow Space along the Ascending Ramus
}

\author{
Hüseyin Akçay, DDS, PhD*, Birkan Tatar, DDS, Fahrettin Kalabalık, DDS, PhD and Murat Ulu, DDS, PhD \\ Faculty of Dentistry, Department of Oral and Maxillofacial Surgery, Izmir Katip Celebi University, Izmir, Turkey
}

\begin{abstract}
The existence of cancellous bone between buccal and lingual cortical plates provides guidance for the desired split plane through the osteotomy lines and interruptions increase the risk of unfavorable fractures during sagittal split ramus osteotomy (SSRO). The aim of this study is to present a rare case of SSRO in the absence of cancellous bone concerning the osteotomy sites, a conservative approach using piezosurgery and bone separators to avoid complications.
\end{abstract}

Keywords

Sagittal Split Ramus Osteotomy, Prognathism, Mandible, Piezosurgery

\section{Introduction}

Sagittal split ramus osteotomy (SSRO) is the most common used technique to correct mandibular deformities. Since the original technique described by Trauner and Obwegeser, several modifications have been reported with the aim of reducing complications. Dal Pont's vertical osteotomy in the molar region, Hunsuck and Epker's short lingual cut modifications are some of the best known [1]. Although it is a very successful and widely accepted method, various complications such as nerve injury, hemorrhage and unfavorable fractures known as bad split patterns can be seen [2]. Precision and experience are important requirements for success.

Unfavorable fractures mainly occur if the osteotomy placed in relatively thinner regions of ramus or where bone marrow space is interrupted between the internal and external cortical bone plates. Wolford, et al. suggested that medial osteotomy placed in cortical bone can result in a bad split [3]. Unfortunately, in rare cases with absence of bone marrow space, osteotomies have to be performed partially or very seldomly totally in cortical bone plate which increases the incidence dramatically.

The aim of this study is to present a rare case of SSRO performed in the absence of bone marrow space bilaterally above the mandibular angle and discuss the strategies and alternative surgical techniques to avoid the complications.

\section{Case Report}

A 24-year-old systematically healthy female patient with severe skeletal Class III malocclusion presented to Izmir Katip Celebi University, Faculty of Dentistry, Department of Oral and Maxillofacial Surgery Clinic for orthognathic surgery following orthodontic phase preparations. After, clinical and ra-

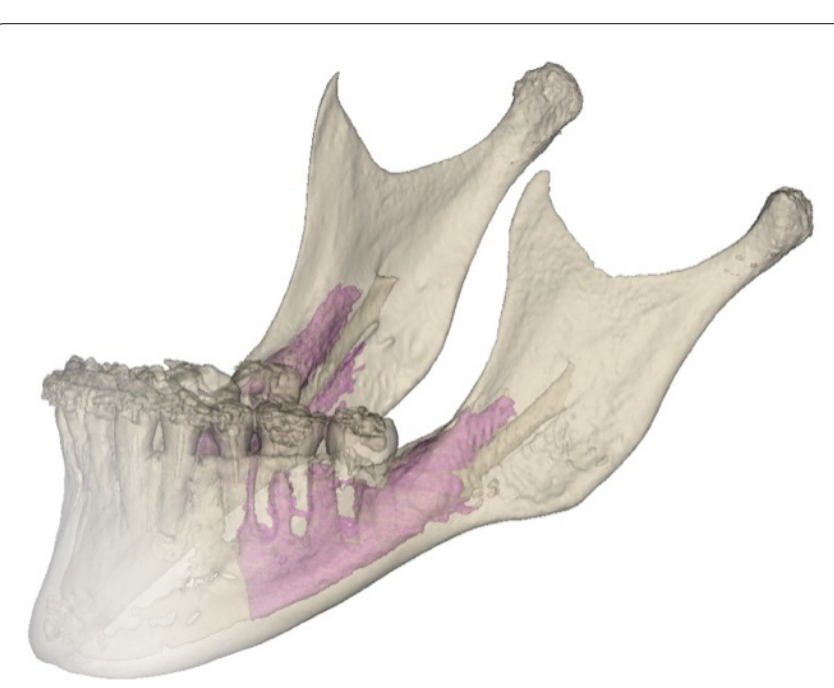

Figure 1: Light purple indicates the cancellous bone structure of posterior mandible. Lateral view shows the absence of bone marrow space above the occlusal level through ascending ramus on each sides.

*Corresponding author: Hüseyin Akçay, DDS, PhD, Assistant Professor, Faculty of Dentistry, Department of Oral and Maxillofacial Surgery, Izmir Katip Celebi University, Aydınlıkevler Mah. CemilMeriç Cad. İçü DişHek, Fak, 35640, Çiğli, İzmir, Turkey, Tel: +905053383882

Accepted: March 24, 2020

Published online: March 26, 2020

Citation: Akçay H, Tatar B, Kalabalık F, et al. (2020) Sagittal Split Ramus Osteotomy in the Absence of Bone Marrow Space along the Ascending Ramus. Archives Oral Maxillofac Surg 3(1):29-32 
Citation: Akçay H, Tatar B, Kalabalık F, et al. (2020) Sagittal Split Ramus Osteotomy in the Absence of Bone Marrow Space along the Ascending Ramus. Archives Oral Maxillofac Surg 3(1):29-32

diological examinations, $6 \mathrm{~mm}$ maxillary advancement with LeFort I and $5 \mathrm{~mm}$ mandibular set-back with SSRO was planned. Written informed consent was obtained from the patient prior to surgery.

The distribution of the cancellous bone at each section was examined with $\mathrm{CBCT}$ imaging. No cancellous bone was found above each mandibular angle along the ascending ramus. Superior border of cancellous bone structure was below the mandibular lingula (ML) $2.8 \mathrm{~mm}$ in the right ramus and $4.6 \mathrm{~mm}$ in the left ramus. So, it was detected that in both sides medial osteotomy had to be totally and sagittal osteotomy had to be partially in cortical bone plate in the absence of bone marrow space that could ease and guide the splitting procedure (Figure 1 and Figure 2).

Under general anesthesia, Le-Forte I maxillary advancement of $6 \mathrm{~mm}$ was first carried out. Then, bilateral sagittal split ramus osteotomy was performed with the short lingual technique described by Hunsuck [1]. Briefly, a horizontal groove was made from the anterior medial cortex of the ramus to a few millimeters posterior to the mandibular foramen just above the ML with a thin Lindemann bur $(1.6 \times 26$ $\mathrm{mm})$.

Due to the thin ramus, vertical and sagittal osteotomies were performed with ultrasonic bone surgery tips attached to piezosurgery device (VarioSurg3, NSK-Nakanishi Inc., Tochigi, Japan) instead of traditional rotating burs and chisels. Split was accomplished by using a Smith bone separator positioned at the sagittal cut and an inferior border separator positioned at the end of the vertical cut. In both sides, IAN was observed in the distal segment when the splitting was done.

After completion of the splitting, the occlusion was fixed with the final splint. The distal and the proximal segments were fixed with a 4-hole mini-plate and screws placed on each side. After removal of the stabilizing splint, the occlusion was checked with the predetermined position. The wound was primarily closed. Nointraoperative or postoperative complications were observed (Figure 3).

\section{Discussion}

SSRO is a technique that could be performed intraorally by separating the lateral and medial cortical bone plates of the ramus above the $\mathrm{ML}$ and just below the mandibular notch, as suggested originally by Obwegeser and Trauner without transfacial approaches and visible scars [1].

Yamamoto, et al. concluded that incidence of neurosensory disturbance was more likely to increase when the width of the bone marrow space is $0.8 \mathrm{~mm}$ or less [4]. The existence
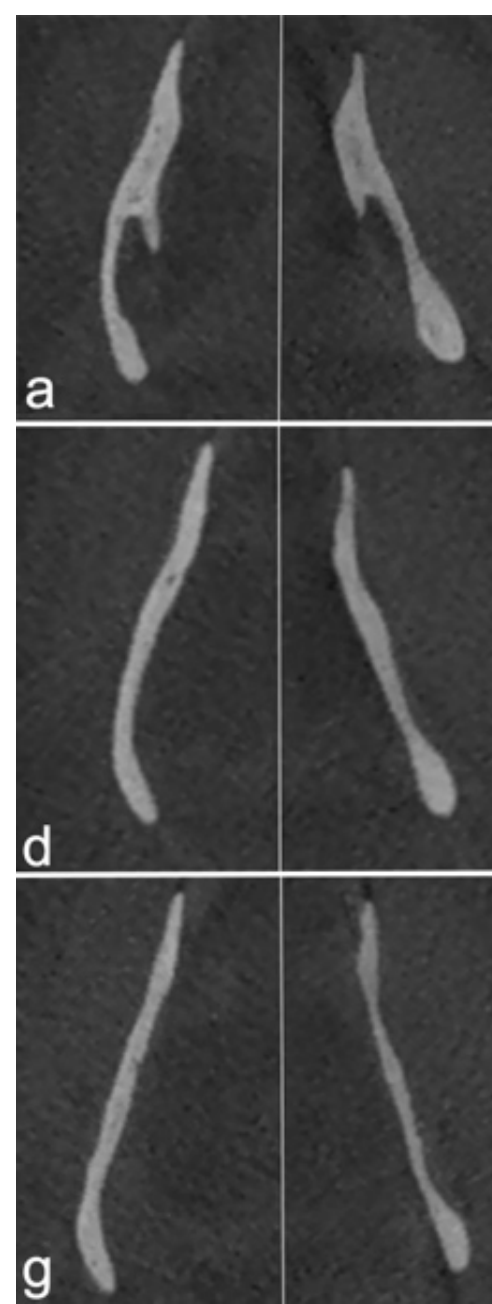
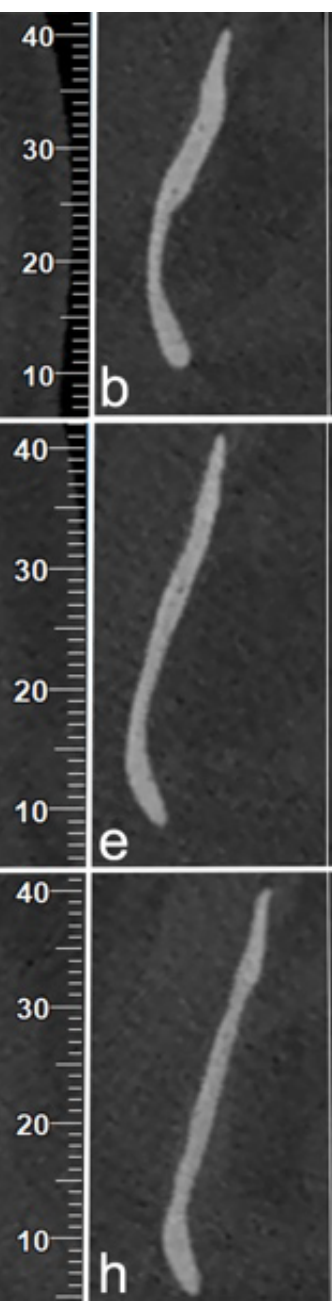
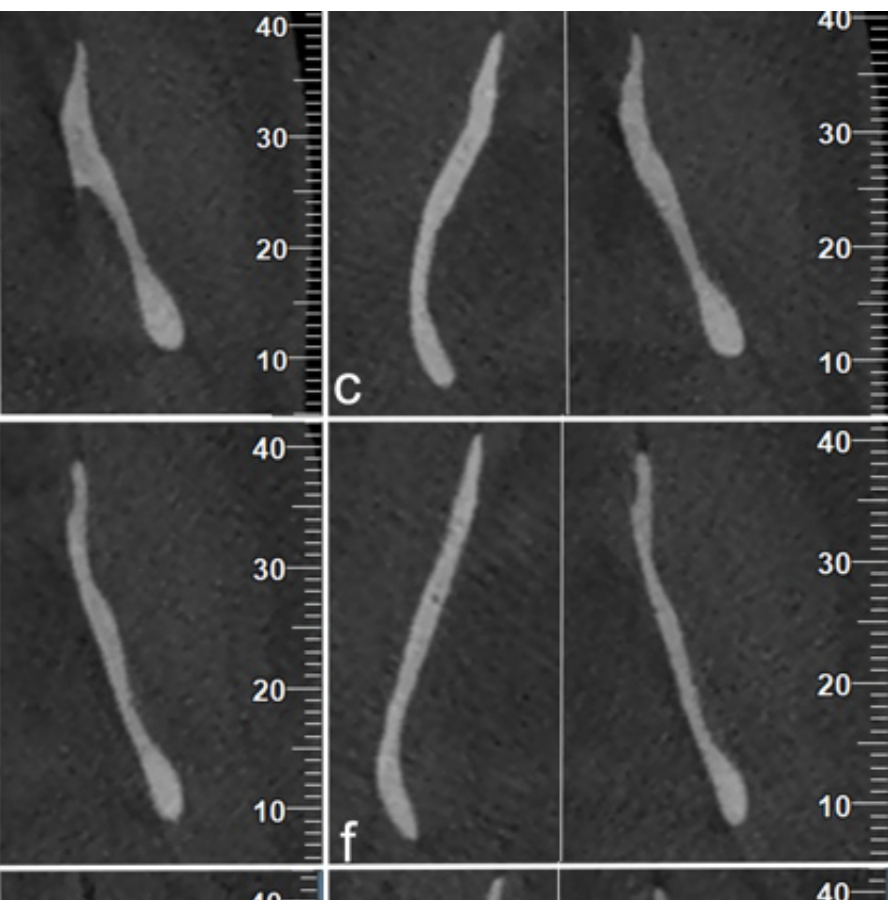

40
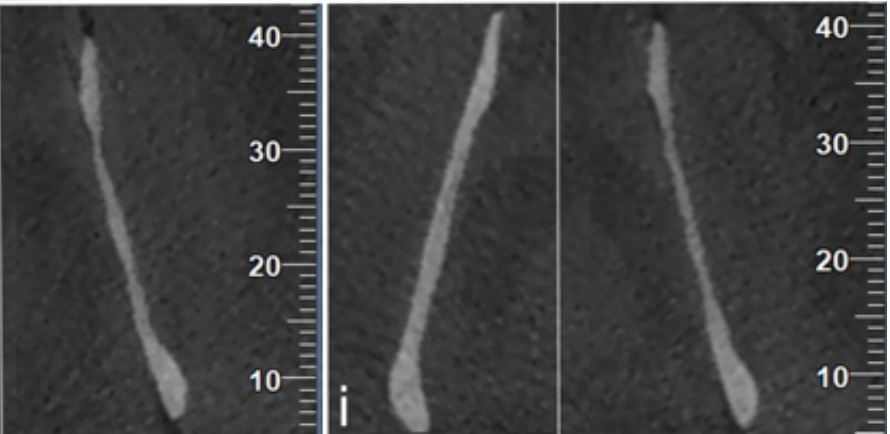

Figure 2: a-i) Axial CBCT sections taken at $2 \mathrm{~mm}$ intervals starting from the lingula. 


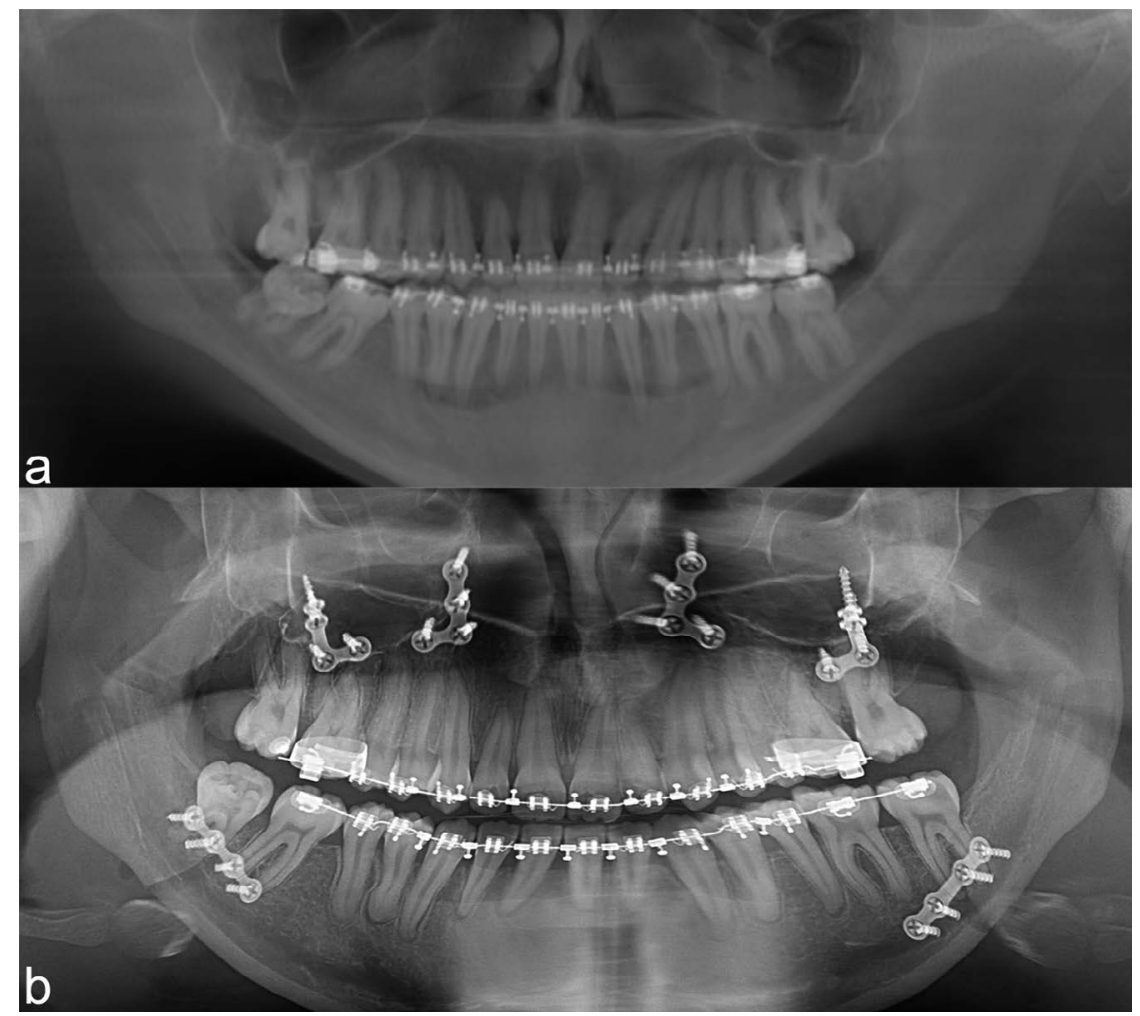

Figure 3: a) Pre-operative; b) Post-operative orthopantomogram images.

of cancellous bone between buccal and lingual cortical plates allows a convenient plane in which a favorable fracture pattern can occur. Medial osteotomy placed in a region without cancellous bone can lead to a bad split. To avoid such complications, some modifications have been reported by many authors. The medial osteotomy should be perpendicular to the ascending ramus at the level of the superior part of the $\mathrm{ML}$ and the posterior end of the osteotomy directed inferiorly a small degree which should be extended at least 3-6 $\mathrm{mm}$ posterior to the ML [3].

There are very few studies about the cancellous bone distribution in ramus mandible of patients with dentoskeletal mandibular deformities. Muto, et al. stated that the cancellous bone structure is quite variable from the ML to the mandibular notch in the ramus and classified the existence and distribution of the bone marrow in their study [5]. The difference was significant between the control group without any dentofacial deformity and skeletal Class III patients group. $22 \%$ of the patients with dentoskeletal Class III deformity had either a separation of cancellous bone at the level of the ML (12\%) or no cancellous bone posterior to the ML $(10 \%)$ which increases the incidence of bad splits. There was no subject having totally cortical bone above the level of mandibular foramen in the study. So, the case we presented above doesn't conform any type in this classification.

The reported incidence of bad splits in SSRO ranges between $0.5 \%$ to $5.5 \%$ [6]. There is a common thought that using chisels for splitting procedure reduces the risk of bad splits whereas the use of separators and splitters without chisels reduce the risk of neurosensory disturbances after SSRO. Mensink, et al. reported the incidence of bad splits $2.0 \%$ in their retrospective study comprised of 427 patients underwent SSRO without chisels [7]. Therefore, using separators and splitters seems to be convenient for reducing the risk of postoperative neurosensory disturbances without increasing the risk of bad split. Also, a systematic review including 14 studies showed that chisel use increases IAN damage in SSRO [8].

In cases with severely narrow mandibular ramus, treatment with only Le Fort I osteotomy or combined with intraoral vertical ramus osteotomy could be considered to prevent complications if a controlled condylar reposition is not a necessity [9]. Intraoral vertical ramus osteotomy is advantageous for reduced operation time, technical simplicity and lower incidence of inferior alveolar nerve injury and bad splits [10]. However, reduced bony interface between segments and necessity of intermaxillary fixation are disadvantages of this technique [11].

Using the piezoelectric bone saw in the same manner as a chisel has advantages compared to conventional chisel osteotomy. Piezosurgery reduces the risk of neural and other surrounding soft tissue damages through a selective cutting, also provides a better vision with less hemorrhage and allows to perform thinner and precise bone cuts compared to traditional systems [12]. Koba, et al. reported significantly reduced operation time, hemorrhage and rate of paresthesia 3 months after osteotomy in the piezosurgery group compared to chisel group in their study including 44 patients [13]. A systematic review including 12 articles and 799 patients showed that the use of piezoelectric surgery leads to significantly better outcome regarding to neurosensory disturbance which was seen in $4.7 \%$ of pa- 
tients who underwent piezoelectric surgery and $61.6 \%$ of patients who underwent surgery with a conventional saw [14]. According to the current studies and their success rates, a combination of piezosurgery, splitters and separators may be considered instead of chisels for high success rates especially for patients with relatively thinner ramus and interrupted bone marrow space.

\section{Conclusion}

Prognathic mandibles are tend to have a thinner ramus with a narrower bone marrow space which increases the risk of complications during and after operation. SSRO concerning the thin ramus in the absence of bone marrow space is very rare but a challenging case requiring precise surgical planning. According to the current studies and their success rates, a combination of piezosurgery, splitters and separators may be considered instead of chisels. Clinical and radiological examinations should be carefully performed to evaluate the anatomic structure before operation and technical modifications should be considered for each case to reduce complications.

\section{Conflict of Interest}

None.

\section{Funding}

Not applicable.

\section{References}

1. Wyatt WM (1997) Sagittal ramus split osteotomy: Literature review and suggested modification of technique. $\mathrm{Br} J$ Oral Maxillofac Surg 35: 137-141.

2. Van Sickels JE (2012) Prevention and management of complications in orthognathic surgery. In: Miloro M, Ghali G, Larsen P, Waite $P$, Peterson's principles of oral and maxillofacial surgery. ( $3^{\text {rd }}$ edn), Shelton, CT: PMPH, USA.

3. Wolford LM, Bennet MA, Rafferty CG (1987) Modification of the mandibular ramus sagittal split osteotomy. Oral Surg Oral Med Oral Pathol 64: 146-155.
4. Yamamoto R, Nakamura A, Ohno K, et al. (2002) Relationship of the mandibular canal to the lateral cortex of the mandibular ramus as a factor in the development of neurosensory disturbance after bilateral sagittal split osteotomy. J Oral Maxillofac Surg 60: 490-495.

5. Muto T, Shigeo K, Yamamoto K, et al. (2003) Computed tomography morphology of the mandibular ramus in prognathism: Effect on the medial osteotomy of the sagittal split ramus osteotomy. J Oral Maxillofac Surg 61: 89-93.

6. Mensink G, Zweers A, Wolterbeek R, et al. (2012) Neurosensory disturbances one year after bilateral sagittal split osteotomy of the mandibula performed with separators: A multi-centre prospective study. J Craniomaxillofac Surg 40: 763-767.

7. Mensink G, Verweij JP, Frank MD, et al. (2013) Bad split during bilateral sagittal split osteotomy of the mandible with separators: A retrospective study of 427 patients. $\mathrm{Br} J$ Oral Maxillofac Surg 51: 525-529.

8. Mensink G, Gooris PJ, Bergsma JE, et al. (2014) Influence of BSSO surgical technique on postoperative inferior alveolar nerve hypoesthesia: A systematic review of the literature. J Craniomaxillofac Surg 42: 976-982.

9. Komori H, Kawanabe N, Kataoka T, et al. (2018) The different effects of intraoral vertical ramus osteotomy (IVRO) and sagittal split ramus osteotomy (SSRO) on mandibular border movement. Cranio 36: 228-233.

10. Ghali GE, Sikes JW Jr (2000) Intraoral vertical ramus osteotomy as the preferred treatment for mandibular prognathism. J Oral Maxillofac Surg 58: 313-315.

11. Wolford LM (2000) The sagittal split ramus osteotomy as the preferred treatment for mandibular prognathism. J Oral Maxillofac Surg 58: 310-312.

12. Vercellotti T, Nevins ML, Kim DM, et al. (2005) Osseous response following resective therapy with piezosurgery. Int J Periodontics Restorative Dent 25: 543-549.

13. Koba A, Tanoue R, Kikuta S, et al. (2018) The usefulness of piezoelectric surgery in sagittal split ramus osteotomy. Kurume Med J 64: 57-63.

14. Silva LF, Carvalho-Reis ENR, Bonardi, JP, et al. (2017) Comparison between piezoelectric surgery and conventional saw in sagittal split osteotomies: A systematic review. Int J Oral Maxillofac Surg 46: 1000-1006. 